\title{
Estratégias no uso da energia luminosa por plantas jovens de Genipa spruceana Steyerm submetidas ao alagamento
}

José Francisco de Carvalho Gonçalves ${ }^{1,2}$, Emanuele Gurgel de Freitas Melo ${ }^{1}$, Carlos Eduardo Moura da Silva ${ }^{1}$, Marciel José Ferreira ${ }^{1}$ e Gilberto Costa Justino ${ }^{1}$

Recebido em 16/05/2011. Aceito em 27/02/2012

\begin{abstract}
RESUMO
(Estratégias no uso da energia luminosa por plantas jovens de Genipa spruceana Steyerm submetidas ao alagamento). Na tentativa de elucidar estratégias de utilização da energia luminosa em plantas jovens de Genipa spruceana Steyerm submetidas ao alagamento, nós investigamos a capacidade de captura e uso de energia luminosa em G. spruceana sob três condições de crescimento: 1- ausência de alagamento (SA), 2- plantas parcialmente alagadas (PA) e 3-plantas totalmente alagadas (TA). Medidas de área foliar específica, teores de pigmentos cloroplastídicos e fluorescência da clorofila $a$ foram feitas em intervalos regulares no período de 90 dias. Todos os parâmetros analisados diminuíram em condições de alagamento (PA e TA). Aos 30 dias, as plantas no tratamento TA sofreram abscisão foliar. Os teores dos pigmentos cloroplastídicos (clorofilas e carotenóides) entre os tratamentos SA e TA diferiram aos 30 dias. Ao passo que, somente foi possível verificar diferenças entre os tratamentos SA e PA aos 90 dias. As plantas submetidas ao alagamento (PA e TA) exibiram alta dissipação de energia de excitação (DI / ABS) indicando limitada eficiência na utilização da energia luminosa. Este fato foi comprovado pelos resultados do índice de desempenho $\left(\mathrm{PI}_{\mathrm{ABS}}\right)$ somente ao fim do período experimental (90 dias). Mas, não foi verificado diferença para $\mathrm{PI}_{\mathrm{ABS}}$ entre os tratamentos aos 30 dias. Portanto, considerando que G. spruceana submetidas ao tratamento TA reduziram seus teores de clorofilas mais rapidamente do que decrescem seus $\mathrm{PI}_{\mathrm{ABS}}$ sugere-se que o fluxo de energia luminosa em plântulas de G. spruceana sob alagamento total, no início, é mais restringido pelo decréscimo na estrutura de captura de luz (diminuição dos pigmentos cloroplastídicos) do que no funcionamento do aparato fotossintético (alterações na eficiência fotoquímica do fotossistema II).
\end{abstract}

Palavras-chave: Plantas alagadas; conteúdo de clorofilas; fluxo fenomenológico; índice de desempenho

\begin{abstract}
(Strategies in the use of light energy by Genipa spruceana Steyerm seedlings subjected to flooding). In an attempt to elucidate strategies in the use of light energy by G. spruceana seedlings subjected to flooding, we investigated the capacity of light capture and use of light energy by G. spruceana in three growing conditions: 1- absence of flooding (SA), 2- partially flooded (PA) and 3- totally flooded (TA). Destructive and non-destructive measurements, such as specific leaf area, chloroplast pigment (chlorophyll and carotenoids) content and fluorescence analyses, were made at regular intervals over a period of 90 days. All parameters decreased in seedlings subjected to flooding. Plants of treatment TA dropped all of their leaves after 30 days of complete submergence. Chloroplast pigment content differed between treatments SA and TA after 30 days from the start of the experiment; whereas SA and PA plants only differed for this variable after 90 days. Plants subjected to flooding (PA and TA) exhibited high dissipation of photochemical de-excitation (DI/ABS), indicating a limited efficiency of light energy use. This fact was proven by the performance index $\left(\mathrm{PI}_{\mathrm{ABS}}\right)$ only in analyses after 90 days, and no significant difference was verified for $\mathrm{PI}_{\mathrm{ABS}}$ among treatments up to 30 days. Therefore, considering that $G$. spruceana seedlings subjected to flooding reduced the chloroplast pigment content more quickly than $\mathrm{PI}_{\mathrm{ABS}}$, we suggest that the light energetic flux in G. spruceana seedlings subjected to flooding, in the beginning, is more restricted to a decrease in the structures that captures light (reduction chlorophyll pigment content) than how the photosynthetic apparatus functions (alterations in photochemical efficiency of photosystem II).
\end{abstract}

Key words: Flooded plants; chlorophyll content; phenomenological fluxes; performance index

\footnotetext{
1 Instituto Nacional de Pesquisas da Amazônia, Coordenação de Pesquisas em Silvicultura tropical, Manaus, AM, Brasil

2 Autor para correspondência: jfc@inpa.gov.br
} 


\section{Introdução}

A maior extensão da floresta amazônica brasileira é constituída por áreas não alagadas (terra firme). No entanto, cerca de $300.000 \mathrm{~km}^{2}$ são ocupadas por florestas inundáveis, classificadas como sendo áreas de várzeas e de igapós (Junk 1997). Ações governamentais têm incorporado artificialmente novas áreas aos $300.000 \mathrm{~km}^{2}$ ocupados por florestas naturalmente inundáveis. Ao longo dos últimos 30 anos, áreas submetidas à inundação pela construção de hidroelétricas têm sido ampliadas na Amazônia (Curua-Una, Balbina, Samuel e Tucuruí) e as futuras hidrelétricas a serem construídas (Altamira, Araguaia, Belo Monte, Jamanxim e Teles Pires) irão alagar aproximadamente $5.000 \mathrm{~km}^{2}$, com a expectativa que as áreas alagadas artificialmente aumentem de forma substancial e avancem sobre áreas de florestas naturais (Tundisi 2007; Fearnside, 2009).

$\mathrm{O}$ fato do surgimento de novas áreas alagadas implica o recobrimento de parte considerável da vegetação às margens dos reservatórios por períodos relativamente longos durante a época das cheias. Assim, devido às alterações no ambiente de crescimento, em particular, nos componentes físicos, químicos e microbiológicos dos solos destas áreas, as plantas tolerantes a tais condições são capazes de desenvolver mecanismos que as permitam suportar a depleção de oxigênio e os eventuais aumentos nas concentrações de compostos tóxicos de caráter redutivo. Os mecanismos pelos quais as plantas toleram a deficiência ou ausência de oxigênio promovida pelo alagamento e suas conseqüências funcionais nas plantas são complexos e envolvem a interação das adaptações fisiológicas, anatômicas e morfológicas, sendo as mais evidentes: expansão foliar; indução de abscisão foliar; senescência; diminuição nas trocas gasosas, ativação do metabolismo anaeróbico, redução do acúmulo de biomassa da raiz, folhas e hastes; hipertrofia de lenticelas e caules, formação de raízes adventícias, aerênquimas e pneumatóforos (Colmer \& Pedersen 2008; Zanandrea et al. 2009; Oliveira \& Joly 2010).

A baixa disponibilidade de oxigênio promove distúrbios metabólicos, em particular, aqueles relacionados à respiração celular nas raízes, e também pode afetar a eficiência fotossintética pela diminuição da produção de energia fotoquímica (Allen et al. 1996). Em estádios mais avançados, esses danos podem ser exteriorizados pelo amarelecimento foliar, devido à redução nos teores de clorofilas resultantes da degradação e/ou decréscimo de sua síntese, como constatado em folhas de várias espécies submetidas a diferentes níveis de inundação (Carvalho \& Ishida 2002; Ishida et al. 2002; Maia \& Piedade 2002; Chen et al. 2005). No entanto, ainda não existe completo esclarecimento sobre a tendência positiva ou negativa do balanço entre síntese e degradação de pigmentos, uma vez que algumas espécies mesmo submetidas ao alagamento não apresentam diminuição nos teores de pigmentos cloroplastídicos (Waldhoff et al. 2002). Estes pigmentos, por sua vez, são determinantes para a absorção de luz e conversão de energia pelas plantas. Desta forma, a captura da energia luminosa e/ou sua dissipação podem ser quantificada analisando-se a fluorescência da clorofila $a$.

A determinação da fluorescência da clorofila $a$ por ser uma técnica rápida, precisa e não destrutiva, tem sido usada para investigar danos causados por vários tipos de estresses sobre o aparato fotossintético (Oliveira et al. 2002; Mielke et al. 2003; Gonçalves et al. 2005; Fernández 2006; Lavinsky et al. 2007; Naumann et al. 2008). Além disso, os parâmetros de fluorescência da clorofila $a$ também têm se mostrado como ferramenta sensível capaz de diagnosticar a redução da assimilação de $\mathrm{CO}_{2}$ e/ou destruição da estrutura fotossintética (Mena-Petite et al. 2003). Sendo o fotossistema II responsável pelo fornecimento de energia para a fotossíntese, a avaliação de sua eficiência mediante medidas da emissão da fluorescência da clorofila $a$ pode ser usada como indicador dos danos causados pelo alagamento em espécies arbóreas. Adicionalmente, verifica-se que contribuições científicas que relacionem as possibilidades de captura e uso de energia em plantas nativas de ambiente alagados são menos freqüentes na literatura. Muito embora, seja conhecido que algumas espécies demonstram potencial para habitar ou mesmo revegetar áreas submetidas a pulsos de alagamentos, dentre estas plantas se destacam espécies do gênero Genipa (Mielke et al. 2003).

$\mathrm{Na}$ Amazônia, em especial, na região de influência da hidroelétrica de Balbina, a espécie Genipa spruceana Steyerm (Rubiaceae) é bastante freqüente, além de ser conhecida a ocorrência desta espécie na Bolívia, Colômbia, Equador, Guiana Francesa, Guiana, Peru, Suriname e Venezuela. Na região marginal da hidroelétrica de Balbina, no Estado do Amazonas, indivíduos de G. spruceana permanecem grande parte do ano sob alagamento, uma vez que, o nível de água da represa é controlado pela vazante para a produção de energia. Adicionalmente, durante as estações de baixa precipitação algumas áreas de influência ficam desprovidas de água. Neste ambiente esta espécie caracteriza-se como planta heliófita, semidecídua e seletiva higrófita. A árvore apresenta porte médio (até $12 \mathrm{~m}$ ) com copa grande e arredondada, folhas simples, opostas, glabras em ambas as faces, ovaladas a elípticas, flores hermafroditas amarelo-ouro contendo cinco pétalas. $\mathrm{O}$ fruto é do tipo baga subglobosa com polpa adocicada. Quando verde o fruto libera uma tintura de cor violeta ou azul escura que serve como corante natural. Sua madeira apresenta importante uso na marcenaria em geral (Ribeiro et al. 1999; Revilla 2002).

Portanto, considerando o cenário de ocorrência e freqüência da G. spruceana em áreas alagadas artificialmente por hidroelétrica, demonstrando adaptação a este tipo de habitat e no intuito de obter melhor entendimento sobre as estratégias fotossintéticas, em particular, aquelas respostas relacionadas à captura e uso da energia luminosa, adotadas pela espécie quando submetidas ao alagamento, nós investigamos a hipótese do "dano controlado" nas plantas de G. spruceana, isto é, embora apresente danos ao sistema 
de captura de luz quando submetida ao alagamento, as mesmas mantêm o funcionamento dos seus fotossistemas, em particular do fotossistema II, mantendo, até certo ponto, as características de captação e uso da energia no processo fotossintético semelhantes aquelas das plantas não alagadas. Este trabalho teve como objetivo investigar as alterações referentes à área foliar específica, aos teores de pigmentos cloroplastídicos (clorofilas $a$ e $b$ e carotenóides) e aos parâmetros relacionados à fluorescência da clorofila $a \mathrm{em}$ plantas jovens submetidas ao alagamento em ambiente controlado.

\section{Material e métodos}

$\mathrm{O}$ experimento foi conduzido na área experimental do campus III (V-8) do Instituto Nacional de Pesquisas da Amazônia (MCTI-INPA), Manaus-AM (3ㅇ's'S e $59^{\circ} 52^{\prime} \mathrm{W}$ ), sob condições controladas de alagamento. As plantas utilizadas no estudo foram oriundas da Reserva Biológica do Uatumã, localizada na área da Usina Hidrelétrica Balbina, Presidente Figueiredo-AM ( $1^{\circ} 55^{\prime} \mathrm{S}$ e $\left.59^{\circ} 28^{\prime} \mathrm{W}\right)$. Oito pontos de coleta de plantas jovens de G. spruceana foram selecionados com distância mínima de 200 m entre os indivíduos amostrados, para evitar pseudo-repetição. As plantas jovens de G. spruceana foram escolhidas de acordo com critérios pré-estabelecidos de uniformidade, de tamanho (altura média de $30 \mathrm{~cm}$ e $6,3 \mathrm{~mm}$ de diâmetro ao nível do coleto - DAC), média de quatro folhas por indivíduo, que apresentassem bom estado fitossanitário e limbo completamente expandido. Os indivíduos selecionados foram coletados no campo, colocados em sacos plásticos $(5 \mathrm{~kg})$ contendo solo da própria área de coleta e levados imediatamente para a área experimental no INPA. No local do experimento, as plantas foram transferidas para vasos $(5 \mathrm{~kg}$ ) completando o substrato de crescimento com mistura de areia e terriço (1:1). Após aclimatação (durante um mês em sol pleno), as plantas foram submetidas a três tratamentos: 1) $\mathrm{SA}=$ sem alagamento; 2) $\mathrm{PA}=$ parcialmente alagado (linha d'água $5 \mathrm{~cm}$ acima do substrato); e 3) $\mathrm{TA}=$ totalmente alagado (linha d'água $5 \mathrm{~cm}$ acima das plantas). As plantas dos três tratamentos foram colocadas dentro de tanques circulares (capacidade de 1000 litros) e organizadas entre si com espaçamento de $40 \mathrm{~cm}$. Para evitar potenciais interferências advindas de propriedades físicas e químicas que pudessem interferir na disponibilidade de oxigênio, a água dos tanques foi trocada a cada três dias. As plantas dos tratamentos PA e TA permaneceram alagadas durante 90 dias.

Nas plantas, selecionadas por sorteio, foram determinados parâmetros destrutivos envolvendo a área foliar específica (AFE), teores de pigmentos cloroplastídicos, bem como, foram medidos na mesma folha os parâmetros não destrutivos relacionados à fluorescência da clorofila $a$. O parâmetro AFE foi determinado pela razão: área foliar / massa foliar seca $\left(70^{\circ} \mathrm{C}\right)$, a partir de discos foliares de área conhecida retirados das folhas, sendo utilizados dez discos foliares por indivíduo (Evans \& Poorter 2001). Para a de- terminação dos pigmentos cloroplastídicos foram coletadas amostras foliares e envolvidas em papel alumínio, acondicionadas em caixas térmicas contendo gelo e conduzidas ao Laboratório de Fisiologia e Bioquímica Vegetal (LFBV) do INPA. No tecido foliar os teores de pigmentos cloroplastídi$\cos$ (clorofilas $a$ e $b$ e dos carotenóides) foram determinados a partir de $0,1 \mathrm{~g}$ de material vegetal fresco e homogeneizado com $10 \mathrm{ml}$ de acetona $(100 \% \mathrm{v} / \mathrm{v})+10 \mathrm{ml}$ de uma solução de acetona $(80 \% \mathrm{v} / \mathrm{v})$ contendo $\mathrm{MgCO}_{3}(0.5 \% \mathrm{p} / \mathrm{v})$, conforme Lichtenthaler \& Wellburn (1983) e calculados, de acordo com equações de MacKinney's descritas por Hendry \& Price (1993). As medidas dos parâmetros relacionados à fluorescência da clorofila $a$ foram feitas em folhas apresentando bom estado fitossanitário e completamente expandidas (maduras), utilizando-se fluorômetro portátil (Handy PEA - v.1,30 - Hansatech Instruments, Norfolk, UK) sempre entre as 8:00 e 12:00 h do dia. As folhas selecionadas foram adaptadas ao escuro durante 30 minutos, utilizando-se clipes apropriados. Após a adaptação, as folhas foram expostas a um pulso saturante de luz ( $3000 \mu \mathrm{mol} \mathrm{m}^{-2} \mathrm{~s}^{-1}$, comprimento de onda de $650 \mathrm{~nm}$, por 1 segundo), obtendo-se as respostas relacionadas aos transientes da fluorescência da clorofila $a$, de acordo com equações do teste JIP. O teste JIP consiste em uma ferramenta computacional desenvolvida para analisar os transientes de fluorescência da clorofila $a$ (OJIP) a partir de diversas reações associadas ao PSII, baseadas na teoria do fluxo de energia em membranas biológicas. Por sua vez, o fluxo de energia através do PSII pode ser discriminado a partir da quantidade de energia absorvida pelos pigmentos cloroplastídicos do complexo antena (ABS) levando à excitação da molécula de clorofila. Parte da energia absorvida é aprisionada pelos centros de reação (TR) para ser convertida à energia redox pela redução do aceptor primário de elétrons $Q_{A}$ à $Q_{A}$ que é então reoxidada à $Q_{A}$ impulsionando o fluxo de elétrons após a $Q_{A}(E T)$ e finalmente levando à assimilação de $\mathrm{CO}_{2}$. A outra parte da energia de excitação é dissipada como calor ou fluorescência (DI) (Strasser et al. 1995). Em seguida, foram calculados os parâmetros do fluxo fenomenológico: $\mathrm{RC} / \mathrm{CS}$ = densidade de centros de reação por seção transversal; $\mathrm{ABS} / \mathrm{CS}$ = número de fotóns absorvidos por seção transversal; DI /CS = energia de dissipação por seção transversal; $\mathrm{TR}_{\mathrm{o}} / \mathrm{CS}=$ taxa máxima de energia bloqueada por seção transversal e; $\mathrm{ET}_{\mathrm{o}} / \mathrm{CS}=$ transporte de elétrons por seção transversal, bem como, os parâmetros das razões de fluxo: $\mathrm{TR}_{\mathrm{o}} / \mathrm{ABS}$ = máxima eficiência quântica do fotossistema II (FSII); DI /ABS = máxima eficiência de excitação não fotoquímica; $\mathrm{ET}_{\mathrm{o}} / \mathrm{TR}_{\mathrm{o}}=$ probabilidade da energia de excitação mover um elétron após a quinona $\mathrm{A}$ e; $\mathrm{ET}_{\mathrm{o}} / \mathrm{ABS}=$ probabilidade de um fóton absorvido mover um elétron após $\mathrm{Q}_{\mathrm{A}}$. Além disso, foram obtidos, também, os valores referente à fluorescência inicial $\left(\mathrm{F}_{\mathrm{o}}\right)$, fluorescência variável $\left(\mathrm{F}_{\mathrm{v}}\right)$, fluorescência máxima $\left(\mathrm{F}_{\mathrm{m}}\right)$, eficiência máxima do fotossistema II $\left(\mathrm{F}_{\mathrm{v}} / \mathrm{F}_{\mathrm{m}}\right)$ e ao índice de desempenho $\left(\mathrm{IP}_{\mathrm{ABS}}\right)$.

O delineamento experimental utilizado foi inteiramente casualizado, com três tratamentos: $\mathrm{SA}=$ sem alagamento; $\mathrm{PA}$ 
$=$ parcialmente alagado; $\mathrm{e} \mathrm{TA}=$ totalmente alagado. Cada tratamento foi composto por cinco repetições (tanques) e cada repetição apresentava cinco indivíduos, somando um total de 75 plantas. Os resultados obtidos foram submetidos ao teste de normalidade Lilliefors e, em seguida, submetidos à análise de variância (ANOVA), sendo posteriormente comparados pelo teste de Tukey $(p<0,05)$. Para um entendimento mais detalhado acerca dos parâmetros obtidos relacionados com o tempo de exposição ao estresse (tempo de alagamento), foi feita análise de regressão quando necessária. O programa utilizado para a realização das análises estatísticas foi o Statistica 6.0 (StatSoft 2003).

\section{Resultados e discussão}

Os valores de AFE diminuíram com o tempo, tanto nas plantas sob efeito de alagamento (PA e TA), quanto nas plantas não alagadas (Controle - SA). Após 90 dias de experimento, as plantas do tratamento $S A$ exibiram redução de $26 \%$ na AFE, enquanto no tratamento PA as plantas reduziram em $42 \%$ sua área foliar e as plantas no tratamento TA não apresentavam mais folhas (Fig. 1). As plantas do tratamento TA começaram, pouco a pouco, perdendo as folhas. A partir dos 30 dias de experimentação, não foi mais possível seguir com as medidas neste tratamento. A diminuição da AFE no tratamento SA pode ser atribuída ao aumento da temperatura dentro dos tanques circulares (aumento de até $5^{\circ} \mathrm{C}$, quando comparado com o ambiente aberto - dados não publicados), uma vez que mesmo sem água as plantas também foram colocadas em tanques circulares de plástico. Por outro lado, no tratamento TA, sugere-se que o efeito seja específico da depleção de oxigênio, uma vez que as plantas estavam completamente submersas. Ainda vale ressaltar que, no tratamento PA, no qual a água foi colocada até 5 $\mathrm{cm}$ acima do nível do substrato, a temperatura interna do tanque também foi elevada de $3-5^{\circ} \mathrm{C}$ acima da temperatura do ambiente aberto (medidas realizadas durante o experimento). Assim, oportunamente ressalta-se que, no tratamento PA, pode ter ocorrido potencialização do estresse (déficit de oxigênio e aumento da temperatura). Genipa spruceana é uma espécie capaz de sobreviver em ambientes alagados, sendo, portanto, em teoria, tolerante ao alagamento (Parolin, 2009; Parolin et al. 2010). Contudo, dentre algumas características de espécies arbóreas intolerantes ao alagamento, incluem-se, senescência e abscisão foliar (Pezeshki 1994; Kozlowski 1997). Estas características foram verificadas em plantas de G. spruceana quando submetidas ao alagamento total (TA), após 30 dias de experimento. Plântulas de Genipa americana submetidas ao alagamento apresentaram diminuição do número de folhas e área foliar, após 43 dias de inundação (Mielke et al. 2003). Do mesmo modo, analisando-se o efeito do alagamento no crescimento de plântulas de G. americana, verificou-se diminuição na área foliar das plantas submetidas ao alagamento e não para AFE (Lavinsky et al. 2007). Por outro lado, em estudo sobre

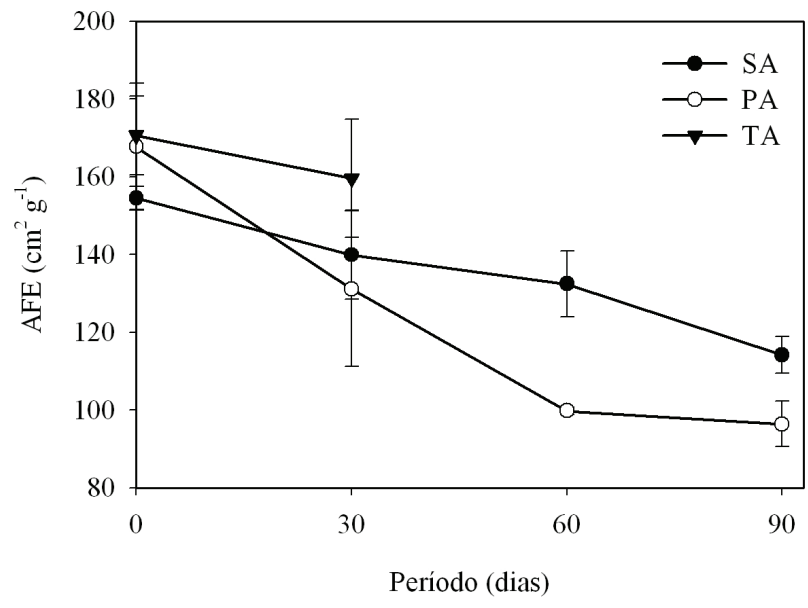

Figura 1. Área foliar específica (AFE) de plantas jovens de Genipa spruceana submetidas ao alagamento. $\mathrm{SA}=$ plantas sem alagamento; $\mathrm{PA}=$ plantas parcialmente alagadas; e $\mathrm{TA}=$ plantas totalmente alagadas. Os valores são médias e as linhas verticais são os desvios padrões $(n=5)$.

mudanças fisiológicas ao alagamento de espécies arbóreas amazônicas (Acosmium nitens, Campsiandra laurifolia, Duroia fusifera, Eschweilera tenuifolia, Pouteria orinocoensis e Symmeria paniculata), verificou-se que a AFE diminuiu com a inundação das plantas (Herrera et al. 2009). Também não foi observado efeito significativo da inundação sobre a AFE de plântulas de Pouteria glomerata, comparado ao tratamento controle (Maurenza et al. 2009). Uma provável explicação para a diminuição da AFE em plantas sob alagamento está relacionada com a redução da translocação de fotossintatos das folhas para outras regiões dreno da planta (Dias-Filho \& Carvalho 2000).

O resultado da análise de variância para os principais parâmetros analisados (AFE, chl $\mathrm{a}_{\mathrm{a}} \mathrm{chl}_{\mathrm{b}}, \mathrm{F}_{\mathrm{v}} / \mathrm{F}_{\mathrm{m}}$ e $\left.\mathrm{PI}_{\mathrm{ABS}}\right)$ demonstrou tanto efeito significativo do nível de alagamento e, também, do tempo, quanto da interação dos mesmos (Tab. 1). Diante desta análise, pode-se observar que os teores de pigmentos cloroplastídicos não diferiram entre os dias observados no tratamento $\mathrm{SA}$, ao passo que, no tratamento $\mathrm{PA}$, somente aos 90 dias de experimento, verificou-se declínio nos teores de $\mathrm{Chl}_{\mathrm{a}}, \mathrm{Chl}_{\mathrm{b}} \mathrm{e} \mathrm{C}_{\mathrm{x}+\mathrm{c}}$, quando comparado com os tempos anteriores ( 0,30 e 60 dias) (Tab. 2). No tratamento TA, aos 30 dias, já se verificou diferença significativa em relação ao tempo zero. Comparando-se os teores de pigmentos entre os tratamentos, verificaram-se, aos 30 dias, baixos valores de $\mathrm{Chl}_{\mathrm{a}}, \mathrm{Chl}_{\mathrm{b}} \mathrm{eC}_{\mathrm{x}+\mathrm{c}}$ no tratamento $\mathrm{TA}$ em relação aos demais tratamentos. Ademais, somente aos 90 dias de experimento, observou-se diferença estatística no tratamento PA, comparada ao tratamento SA. De modo geral, reduções dos teores foliares de pigmentos cloroplastídicos têm sido verificadas em grande número de espécies sob condição de alagamento (Carvalho \& Ishida 2002; Ishida et al. 2002; Maia \& Piedade 2002; Chen et al. 2005; Waldhoff et al. 2002). Provavelmente, a redução nos teores de clorofilas nas plantas sob alagamento, de certa forma, deva estar relacionada com a diminuição na quantidade de $\mathrm{O}_{2}$ disponível para as 
Tabela 1. Resultado da ANOVA (valores $F$ ) para os efeitos e interações dos parâmetros: área foliar específica (AFE), clorofila $a\left(\mathrm{Chl}_{\mathrm{a}}\right)$, clorofila $b$ (Chl $)$, carotenóides $\left(\mathrm{C}_{\mathrm{x}+\mathrm{c}}\right)$, eficiência quântica do fotossistema II $\left(\mathrm{F}_{\mathrm{v}} / \mathrm{F}_{\mathrm{m}}\right)$ e índice de desempenho $\left(\mathrm{PI}_{\mathrm{ABS}}\right)$ de plantas jovens de Genipa spruceana $(n=5)$. ${ }^{*}=$ $p<0,05 ;{ }^{* *}=p<0,01 ;{ }^{* *}=p<0,001 ;$ e ns $=$ não significativo.

\begin{tabular}{lccccccc}
\hline Efeito & $\mathrm{GL}$ & $\mathrm{AFE}$ & $\mathrm{Chl}_{\mathrm{a}}$ & $\mathrm{Chl}_{\mathrm{b}}$ & $\mathrm{C}_{\mathrm{x}+\mathrm{c}}$ & $\mathrm{F}_{\mathrm{v}} / \mathrm{F}_{\mathrm{m}}$ & $\mathrm{PI}_{\mathrm{ABS}}$ \\
\hline Alagamento & 2 & $6,24^{*}$ & $15,48^{* * *}$ & $11,78^{* *}$ & $\mathrm{~ns}$ & $7,74^{* *}$ & $5,02^{*}$ \\
Tempo & 3 & $15,26^{* * *}$ & $34,45^{* * *}$ & $29,77^{* * *}$ & $13,99^{* *}$ & $34,34^{* * *}$ & $4,92^{* *}$ \\
Interação & 11 & $3,69^{*}$ & $6,75^{* *}$ & $4,27^{*}$ & $\mathrm{~ns}$ & $22,76^{* * *}$ & $2,90^{*}$ \\
\hline
\end{tabular}

Tabela 2. Teores de pigmentos cloroplastídicos $\left(\mu \mathrm{mol} \mathrm{g}^{-1}\right)$ de plantas jovens de Genipa spruceana submetidas ao alagamento. SA = plantas sem alagamento; $\mathrm{PA}=$ plantas parcialmente alagadas; $\mathrm{TA}=$ plantas totalmente alagadas; $\mathrm{Chl}=$ clorofila $a ; \mathrm{Chl}_{\mathrm{b}}=$ clorofila $b ; \mathrm{e} \mathrm{C}_{\mathrm{x}+\mathrm{c}}=$ carotenóides. Os valores são médias \pm desvios padrões. Médias seguidas da mesma letra maiúscula na vertical (dias) e da mesma letra minúscula na horizontal (tratamentos), não diferem significativamente pelo teste de Tukey $(p<0,05)(n=5){ }^{*}=$ plantas sem folhas.

\begin{tabular}{|c|c|c|c|c|}
\hline \multirow{2}{*}{ Parâmetro } & \multirow{2}{*}{ Período (dias) } & \multicolumn{3}{|c|}{ Tratamento } \\
\hline & & SA & $\mathrm{PA}$ & $\mathrm{TA}$ \\
\hline \multirow{4}{*}{$\mathrm{Chl}_{\mathrm{a}}$} & 0 & $1,55 \pm 0,31 \mathrm{Aa}$ & $1,54 \pm 0,23 \mathrm{Aa}$ & $1,48 \pm 0,21 \mathrm{Aa}$ \\
\hline & 30 & $1,32 \pm 0,17 \mathrm{Aa}$ & $1,21 \pm 0,07 \mathrm{Aa}$ & $0,63 \pm 0,06 \mathrm{Bb}$ \\
\hline & 60 & $1,48 \pm 0,21 \mathrm{Aa}$ & $1,23 \pm 0,04 \mathrm{Aa}$ & * \\
\hline & 90 & $1,44 \pm 0,10 \mathrm{Aa}$ & $0,72 \pm 0,02 \mathrm{Bb}$ & * \\
\hline \multirow{4}{*}{$\mathrm{Chl}_{\mathrm{b}}$} & 0 & $0,57 \pm 0,13 \mathrm{Aa}$ & $0,56 \pm 0,09 \mathrm{Aa}$ & $0,54 \pm 0,08 \mathrm{Aa}$ \\
\hline & 30 & $0,47 \pm 0,05 \mathrm{Aa}$ & $0,41 \pm 0,04 \mathrm{Ba}$ & $0,25 \pm 0,03 \mathrm{Bb}$ \\
\hline & 60 & $0,52 \pm 0,08 \mathrm{Aa}$ & $0,46 \pm 0,03 \mathrm{ABa}$ & * \\
\hline & 90 & $0,54 \pm 0,07 \mathrm{Aa}$ & $0,28 \pm 0,00 \mathrm{Cb}$ & * \\
\hline \multirow{4}{*}{$C_{x+c}$} & 0 & $0,75 \pm 0,17 \mathrm{Aa}$ & $0,71 \pm 0,18 \mathrm{Aa}$ & $0,71 \pm 0,12 \mathrm{Aa}$ \\
\hline & 30 & $0,65 \pm 0,05 \mathrm{Aa}$ & $0,61 \pm 0,09 \mathrm{Aa}$ & $0,37 \pm 0,03 \mathrm{Bb}$ \\
\hline & 60 & $0,65 \pm 0,11 \mathrm{Aa}$ & $0,65 \pm 0,04 \mathrm{Aa}$ & * \\
\hline & 90 & $0,62 \pm 0,07 \mathrm{Aa}$ & $0,44 \pm 0,01 \mathrm{Bb}$ & * \\
\hline
\end{tabular}

plantas em ambientes alagados, ocasionando restrições no metabolismo, como por exemplo, redução das atividades da enzima redutase do nitrato, prejudicando a absorção e o transporte de íons, tendo como conseqüência, redução nos teores de nitrogênio na parte aérea da planta, que por sua vez está intimamente relacionado com os teores de clorofilas (Drew 1999; Netto et al. 2005). O fato da ausência de diferença significativa nos teores de clorofilas entre os tratamentos SA e PA até 60 dias de experimento, pode ser devido às plantas suportarem a síntese de clorofilas e a produção de ATP, concentrados no fotossistema I (FSI), enquanto os complexos coletores de luz são degradados lentamente (Lippert et al. 2001).

Quanto à fluorescência da clorofila $a$, verificou-se relações significativas para as plantas dos tratamentos PA e TA em função do tempo de exposição ao alagamento (30 e 90 dias para os tratamentos TA e PA, respectivamente) para maioria dos parâmetros, indicando mudanças quanto ao uso da energia luminosa pela plantas sob efeito de alagamento (Tab. 3). No tratamento SA, somente os parâmetros $\mathrm{DI}_{\mathrm{o}} / \mathrm{CS}$ e RC/CS exibiram, ainda que fraca, relação com o tempo de experimentação. Provavelmente, a exposição à alta incidência luminosa pode ter contribuído para redução na densidade de centros de reação (por seção transversal), uma vez que, se verificou aumentos na dissipação de energia pelas plantas em SA. A dissipação de energia absorvida ou máximo rendimento quântico de energia não-fotoquímica (DI /ABS) foi altamente relacionada com o alagamento $\left(\mathrm{r}^{2}=\right.$ 0,90 e 0,77 nos tratamentos PA e TA, respectivamente). Contudo, essa dissipação pode está relacionada mais fortemente ao efeito da degradação de clorofilas por consequência do efeito do alagamento. Os valores de $\mathrm{PI}_{\mathrm{ABS}}$ nas plantas dos tratamentos PA e TA diminuíram em relação ao tempo de exposição ao alagamento, quando comparados com SA (Fig. 2 e Tab. 3). No presente experimento, valores muito baixos da razão $\mathrm{F}_{\mathrm{v}} / \mathrm{F}_{\mathrm{m}}$ foram observados no tratamento $\mathrm{TA}$ até os 60 dias de experimentação (Fig. 3), assim como foi diagnosticado em outras plantas submetidas a tratamentos de inundação (Ishida et al. 2002; Fernández 2006). Em Symmeria paniculata esse parâmetro não foi afetado pelo período de inundação, havendo apenas diferença quanto à idade das folhas (Waldhoff et al. 2002). Adicionalmente, em plantas de Genipa americana submetidas ao alagamento, verificou-se que aos 14 dias após o alagamento houve aumento de $187 \%$ no quenching não-fotoquímico $\left(q_{\mathrm{n}}\right)$, mas não houve diferença estatística em $\mathrm{F}_{\mathrm{v}} / \mathrm{F}_{\mathrm{m}}$ (Mielke et al. 2003). 


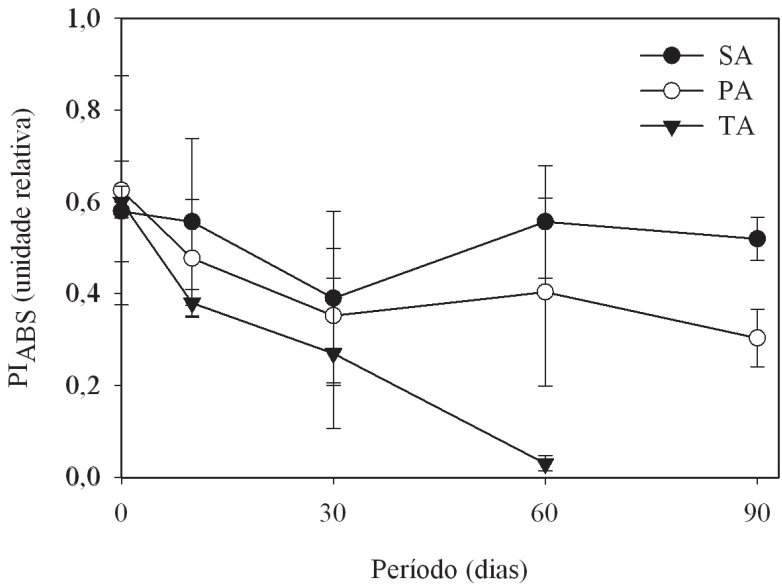

Figura 2. Índice de desempenho $\left(\mathrm{PI}_{\mathrm{ABS}}\right)$ de plantas jovens de Genipa spruceana submetidas ao alagamento. $\mathrm{SA}=$ plantas sem alagamento; $\mathrm{PA}$ $=$ plantas parcialmente alagadas; e TA = plantas totalmente alagadas. Os valores são médias e as linhas verticais são os desvios padrões $(n=5)$.

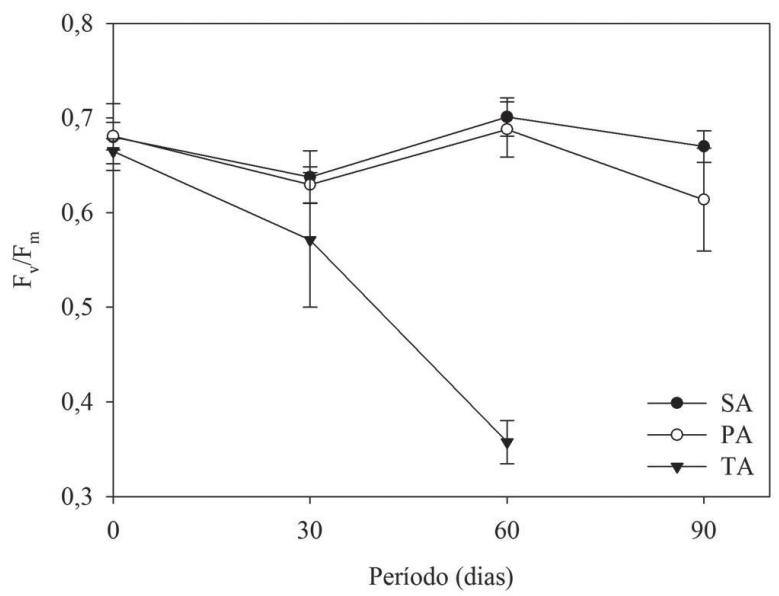

Figura 3. Razão $\mathrm{F}_{\mathrm{v}} / \mathrm{F}_{\mathrm{m}}$ de plantas jovens de Genipa spruceana submetidas ao alagamento. $\mathrm{SA}=$ plantas sem alagamento; $\mathrm{PA}$ = plantas parcialmente alagadas; e TA = plantas totalmente alagadas. Os valores são médias e as linhas verticais são os desvios padrões $(n=5)$.

Tabela 3. Relação dos parâmetros da fluorescência da clorofila $a$ (ver metodologia) em função do tempo (dias) em plantas jovens de Genipa spruceana submetidas ao alagamento. $\mathrm{SA}=$ plantas sem alagamento; $\mathrm{PA}=$ plantas parcialmente alagadas; $\mathrm{TA}=$ plantas totalmente alagadas; $\mathrm{r}^{2}=$ coeficiente de determinação; $\mathrm{b}=$ fator de curvatura; $p=$ probabilidade; e ns = não significativo $(n=5)$.

\begin{tabular}{|c|c|c|c|c|c|c|c|c|c|}
\hline \multirow{3}{*}{ Parâmetro } & \multicolumn{9}{|c|}{ Tratamento } \\
\hline & \multicolumn{3}{|c|}{ SA } & \multicolumn{3}{|c|}{ PA } & \multicolumn{3}{|c|}{$\mathrm{TA}$} \\
\hline & $\mathrm{r}^{2}$ & $\mathrm{~b}$ & $p$ & $\mathrm{r}^{2}$ & $\mathrm{~b}$ & $p$ & $\mathrm{r}^{2}$ & $\mathrm{~b}$ & $p$ \\
\hline $\mathrm{F}_{\mathrm{o}}$ & & ns & & & ns & & 0,72 & $-1,311$ & $<0,01$ \\
\hline$F_{v}$ & & ns & & 0,73 & $-3,039$ & $<0,001$ & 0,79 & $-11,46$ & $<0,05$ \\
\hline $\mathrm{F}_{\mathrm{m}}$ & & ns & & 0,67 & $-3,112$ & $<0,001$ & 0,83 & $-12,771$ & $<0,01$ \\
\hline $\mathrm{F}_{\mathrm{v}} / \mathrm{F}_{\mathrm{m}}$ & & ns & & 0,90 & $-0,001$ & $<0,001$ & 0,77 & $-0,004$ & $<0,01$ \\
\hline $\mathrm{DI}_{\mathrm{o}} / \mathrm{ABS}$ & & ns & & 0,90 & 0,001 & $<0,001$ & 0,77 & 0,004 & $<0,05$ \\
\hline $\mathrm{TR}_{\mathrm{o}} / \mathrm{ABS}$ & & ns & & 0,90 & $-0,001$ & $<0,001$ & 0,77 & $-0,004$ & $<0,01$ \\
\hline $\mathrm{ET}_{\mathrm{o}} / \mathrm{ABS}$ & & ns & & 0,21 & $-0,001$ & $<0,001$ & 0,53 & $-0,002$ & $<0,05$ \\
\hline $\mathrm{ET}_{\mathrm{o}} / \mathrm{TRo}$ & & ns & & & ns & & 0,40 & $-0,002$ & $<0,05$ \\
\hline $\mathrm{ABS} / \mathrm{CS}$ & & ns & & & ns & & 0,72 & $-1,311$ & $<0,01$ \\
\hline $\mathrm{DI}_{\mathrm{o}} / \mathrm{CS}$ & 0,41 & 0,066 & $<0,001$ & 0,97 & 0,346 & $<0,001$ & 0,65 & 1,635 & $<0,05$ \\
\hline $\mathrm{TR}_{\mathrm{o}} / \mathrm{CS}$ & & ns & & 0,58 & $-0,419$ & $<0,001$ & 0,84 & $-2,947$ & $<0,01$ \\
\hline $\mathrm{ET}_{\mathrm{o}} / \mathrm{CS}$ & & ns & & 0,28 & $-0,252$ & $<0,001$ & 0,66 & $-1,261$ & $<0,05$ \\
\hline $\mathrm{RC} / \mathrm{CS}$ & 0,26 & $-0,133$ & $<0,001$ & 0,99 & $-0,500$ & $<0,001$ & 0,94 & $-1,100$ & $<0,01$ \\
\hline $\mathrm{PI}_{\mathrm{ABS}}$ & & ns & & 0,68 & $-0,003$ & $<0,01$ & 0,57 & $-0,004$ & $<0,05$ \\
\hline
\end{tabular}

Porém, em termos absolutos, a razão $\mathrm{F}_{\mathrm{v}} / \mathrm{F}_{\mathrm{m}}$ foi menor que nas plantas controle, assim como, observado em estudo de submersão e recuperação de plantas jovens de Alternanthera philoxeroides e Hemarthria altissima (Lavinsky et al. 2007; Luo et al. 2011). O declínio nos valores da razão $\mathrm{F}_{\mathrm{v}} / \mathrm{F}_{\mathrm{m}}$ indica diminuição na capacidade do PSII reduzir o aceptor primário da quinona A (Panda et al. 2006), o que pode ser evidenciado com a elevação dos valores de DI / ABS, efeito este também observado neste trabalho com G. spruceana . Em estudos sobre exposição de plantas à deficiência hídrica (Oukarroum et al. 2007) ou exposição a diferentes intensidades de irradiância (Gonçalves et al. 2010), o parâmetro
$\mathrm{PI}_{\mathrm{ABS}}$ se mostrou ferramenta mais indicada para verificar estresse em planta do que a razão $\mathrm{F}_{\mathrm{v}} / \mathrm{F}_{\mathrm{m}}$, uma vez que permite avaliar a eficiência do uso da energia mais claramente pelo fato de relacionar a densidade dos centros de reações e a probabilidade da energia ir além da quinona A (Gonçalves et al. 2007), fato claramente observado neste experimento para o parâmetro $\mathrm{PI}_{\mathrm{ABS}}$ na comparação entre os tratamentos aplicados (Fig. 3). O valor exibido pelo tratamento SA é semelhante ao apresentado por plantas jovens de B. excelsa, o qual refletiu melhor desempenho desta espécie crescendo em área degradada submetida à adubação verde (Ferreira et al. 2009). Por outro lado, baixos valores de $\mathrm{PI}_{\mathrm{ABS}}$ indica- 
ram pior desempenho de $B$. excelsa sob adubação química convencional a semelhança de G. spruceana submetida ao alagamento. Tecnicamente, este resultado amplia o alcance de uso desta metodologia também para selecionar plantas tolerantes ao alagamento com mais confiança no alcance desta variável.

Portanto, considerando que plantas de G. spruceana submetidas ao tratamento TA reduziram seus teores de pigmentos cloroplastídicos mais rapidamente do que a eficiência fotoquímica (p.ex.: $\mathrm{PI}_{\mathrm{ABS}}$ ), sugere-se que o fluxo de energia luminosa em plântulas de G. spruceana sob alagamento total, pode ser mais fortemente restringido pelo decréscimo na estrutura de captura de luz, devido a diminuição das concentrações das clorofilas ( $a$ e $b$ ) e dos carotenóides, do que pelas alterações sofridas no funcionamento do aparato fotossintético, sendo a eficiência fotoquímica do fotossistema II menos afetada comparativamente. Diante dos resultados apresentados neste trabalho, o entendimento da utilização da energia luminosa por plantas de G. spruceana quando submetidas ao alagamento, em particular, no que diz respeito à fase fotoquímica da fotossíntese, pode-se assumir que a estratégia de tolerância de plantas que usa como fator determinante a captura da energia luminosa (manutenção das concentrações de pigmentos cloroplastídicos), para G. spruceana não é preponderante. Enfatizando-se que a referida espécie deve usar como estratégia a eficiência na utilização da energia. Do ponto vista de mecanismo de tolerância, é provável que estes dois eventos juntos (modulação da captura e do uso da energia luminosa) podem configurar o início da tolerância desta espécie a ambientes temporariamente alagados, uma vez que mesmo após 90 dias experimentais a taxa de sobrevivência foi igual a $100 \%$, verificando-se plantas alagadas com suas partes aéreas verdes, mesmo sem folhas, com lançamentos de folhas novas após o término do experimento (dados não publicados). No cenário da ecologia de populações dessa espécie, o fato de G. spruceana ser capaz de manter o funcionamento do aparato fotossintético sob ambiente inundado pode constituir indicativo de maior plasticidade ecofisiológica dessa espécie implicando maior capacidade de distribuição da mesma em áreas alagadas e de transição entre platô e baixio em toda bacia Amazônica, credenciado-a para inclusão na lista de árvores nativas da Amazônia aptas à sobrevivência em ambientes temporariamente inundados, se prestando ainda para recuperação áreas degradadas pela construção de hidroelétricas.

\section{Agradecimentos}

Os autores desta pesquisa agradecem ao Instituto Nacional de Pesquisas da Amazônia (MCTI-INPA) pelo suporte logístico, ao Laboratório de Fisiologia e Bioquímica Vegetal pela infra-estrutura para realização dos experimentos e ao CNPq pelas concessões das bolsas de (mestrado, DTI e produtividade), assim como pelo suporte financeiro.

\section{Referências bibliográficas}

Allen, J.A.; Pezensky, S.R. \& Chambers, J.L. 1996. Interaction of flooding and salinity stress on balcypress (Taxodium distichum). Tree Physiology 16: 307-313.

Carvalho, C.J.R. \& Ishida, F.Y. 2002. Resposta de pupunheiras (Bactris gasipaes Kunth) jovens ao alagamento. Pesquisa Agropecuária Brasileira 37(9): 1231-1237.

Chen, H.; Qualls, R.G. \& Blank, R.R. 2005. Effect of soil flooding on photosynthesis, carbohydrate partitioning and uptake in the invasive exotic Lepidium latifolium. Aquatic Botany 82: 250-268.

Colmer, T.D. \& Pedersen, O. 2008. Underwater photosynthesis and respiration in leaves of submerged wetland plants: gas films improve $\mathrm{CO}_{2}$ and $\mathrm{O}_{2}$ exchange. New Phytologist 177: 918-926.

Dias-Filho, M.B. \& Carvalho, C.J.R. 2000. Physiological and morphological responses of Brachiaria spp. to flooding. Pesquisa Agropecuária Brasileira 35(10): 1959-1966.

Drew, M.C. 1999. Oxygen deficiency and root metabolism injury and acclimatation under hypoxia and anoxia. Annual Review Plant Physiology Plant Molecular Bology 48: 223-250.

Evans, J.R. \& Poorter, H. 2001. Photosynthetic acclimation of plants to growth irradiance: the relative importance of specific leaf area and nitrogen partitioning in maximizing carbon gain. Plant, Cell and Environment 24: 755-767.

Fearnside, P.M. 2009. As hidrelétricas de Belo Monte e Altamira (Babaquara) como fonte de gases do efeito estufa. Novos Cadernos NAEA 12(2): 5-56.

Ferreira, M.J.; Gonçalves, J.F.C. \& Ferraz, J.B.S. 2009. Photosynthetic parameters of young Brazil nut (Bertholletia excelsa H. B.) plants subjected to fertilization in a degraded area in Central Amazonia. Photosynthetica 47(4): 616-620.

Fernández, M.D. 2006. Changes in photosynthesis and fluorescence in response to flooding in emerged and submerged leaves of Pouteria orinocoensis. Photosynthetica 44(1): 32-38.

Gonçalves, J.F.C. \& Santos Junior, U.M. 2005. Utilization of the chlorophyll $a$ fluorescence techniques as a tool for selecting tolerant species to environments of high irradiance. Brazilian Journal of Plant Physiology 17(3): 307-313.

Gonçalves, J.F.C.; Santos Júnior, U.M.; Nina Júnior, A.R. \& Chevreuil, L.R. 2007. Energetic flux and performance index in copaíba (Copaifera multijuga Hayne) and mahogany (Swietenia macrophylla King) seedlings grown under two irradiance environments. Brazilian Journal Plant Physiology 19(3): 171-184.

Gonçalves, J.F.C.; Silva, C.E.M.; Guimarães, D.G. \& Bernardes, R.S. 2010 Análise dos transientes da fluorescência da clorofila $a$ de plantas jovens de Carapa guianensis e de Dipteryx odorata submetidas a dois ambientes de luz. Acta Amazonica 40(1): 89-98.

Hendry, G.A.F. \& Price, A.H. 1993. Stress indicators: chlorophylls and carotenoids. Pp. 148-152. In: Hendry, G.A.F. \& Grime, J.P. (Eds). Methods in comparative plant ecology. London, Chapman \& Hall.

Herrera, A.; Escala, M. \& Rengifo, E. 2009. Leaf anatomy changes related to physiological adaptations to flooding in Amazonian tree species. Brazilian Journal Plant Physiology 21(4): 301-308.

Ishida, F.Y.; Oliveira, L.E.M.; Carvalho, C.J.R. \& Alves, J.D. 2002. Efeito da inundação parcial e total sobre o crescimento, teor de clorofila e fluorescência de Setaria anceps e Paspalum repens. Ciência e Agrotecnologia 26(6): 1152-1159.

Junk, W.J. 1997. General aspects of floodplain ecology with special reference to Amazonian floodplains. Ecological Studies 126: 3-20.

Kozlowski, T.T. 1997. Responses of woody plants to flooding and salinity. Tree Physiology Monograph 1: 1-29.

Lavinsky, A.O.; Sant 'Ana, C.S.; Mielke, M.S.; Almeida, A.F.; Gomes, F.P.; França, S. \& Silva, D.C. 2007. Effects of light availability and soil flooding on growth and photosynthetic characteristics of Genipa americana L. seedlings. New Forests 34: 41-50.

Lichtenthaler H.K. \& Wellburn A.R. 1983. Determination of total carotenoids and chlorophyll $a$ and $b$ of leaf extracts in different solvents. Biochemical Society Transactions 11: 591-603. 
Lippert, I.; Rolletschek, H. \& Kohl, J.-G. 2001. Photosynthetic pigments and efficiencies of two Phragmites australis stands in different nitrogen availabilities. Aquatic Botany 69: 359-365.

Luo, F.L.; Nagel, K.A.; Scharr, H.; Zeng, B.; Schurr, U. \& Matsubara, S. 2011. Recovery dynamics of growth, photosynthesis and carbohydrate accumulation after de-submergence: a comparison between two wetland plants showing escape and quiescence strategies. Annals of Botanny 107: 49-63.

Maia, L.A. \& Piedade, M.T. 2002. Influência do pulso de inundação na fenologia foliar e conteúdo de clorofila em duas espécies da floresta de igapó na Amazônia Central, Brasil. Acta Amazonica 32(1): 55-64.

Maurenza, D.; Marenco, R.A. \& Piedade, M.T.F. 2009. Efeito da inundação de longa duração sob o crescimento de Pouteria glomerata (Sapotaceae), uma arbórea da várzea da Amazônia Central. Acta Amazonica 39(3): 519-526.

Mena-Petite, A.; Robredo, A.; Ascalde, S.; Duñabeitia, M.K.; GonzálezMoro, B.; Lacuesta, M. \& Muñoz-Rueda, A. 2003. Gas exchange and chlorophyll fluorescence responses of Pinus radiata D. Don seedlings during and after several storage regimes and their effects on postplanting survival. Trees 17: 133-143.

Mielke, M.S.; Almeida, A.F.; Gomes, F.P.; Aguilar, M.A.G. \& Mangabeira, P.A.O. 2003. Leaf gas exchange, chlorophyll fluorescence and growth responses of Genipa americana seedlings to soil flooding. Environment and Experimental Botany 50: 221-231.

Naumann, J.C.; Young, D.R. \& John, E. 2008. Leaf chlorophyll fluorescence, reflectance, and physiological response to freshwater and saltwater flooding in the evergreen shrub, Myrica cerifera. Environmental and Experimental Botany 63: 402-409.

Netto, A.T.; Campostrini, E.; Oliveira, J.G. \& Bressan-Smith, R.E. 2005. Photosynthetic pigments, nitrogen, chlorophyll $a$ fluorescence and SPAD502 readings in coffee leaves. Scientia Horticulturae 104: 199-209.

Oliveira, J.G.; Alves, P.L.C.A. \& Magalhães, A.C. 2002. The effect of chilling on the photosynthetic activity in coffee (Coffea arabica L.) seedlings. The protective action of chloroplastid pigments. Brazilian Journal of Plant Physiology 14(2): 95-104.

Oliveira, V.C. \& Joly, C.A. 2010. Flooding tolerance of Calophyllum brasiliense Camb. (Clusiaceae): morphological, physiological and growth responses. Trees 24: 185-193.
Oukarroum, A.; El Madidi, S.; Schansker, G. \& Strasser, R.J. 2007. Probing the responses of barley cultivars (Hordeum vulgare L.) by chlorophyll $a$ fluorescence OLKJIP under drought stress and re-watering. Environmental and Experimental Botany 60: 438-446.

Panda, D.; Rao, D.N.; Sharma, S.G.; Strasser, R.J. \& Sarkar, R.K. 2006. Submergence effects on rice genotypes during seedling stage: Probing of submergence driven changes of photosystem 2 by chlorophyll a fluorescence induction O-J-I-P transients. Photosynthetica 44(1): 69-75.

Parolin P. 2009. Submerged in darkness: adaptations to prolonged submergence by woody species of the Amazonian Floodplains. Annals of Botany 103: 359-376.

Parolin, P.; Lucas, C; Piedade, M.T.F.; Wittmann, F. 2010. Drought responses of flood-tolerant trees in Amazonian floodplains. Annals of Botany 105: $129-139$.

Pezeshki, S.R. 1994. Plant response to flooding. Pp. 289-321. In: Wilkinson, R.E. (Ed.). Plant Environment Interactions. New York, Marcel Dekker.

Revilla, J. 2002. Plantas úteis da bacia amazônica. Manaus, SEBRAEAM/INPA.

Ribeiro, J.E.L.S.; Hopkins, M.J.G.; Vicentini, A.; Sothers, C.A.; Costa, M.A.S.; Brito, J.M.; Souza, M.A.D.; Martins, L.H.P.; Lohmann, L.G.; Assunção, P.A.C.L.; Pereira, E.C.; Silva, C.F.; Mesquita, M.R. \& Procópio, L.C. 1999. Flora da Reserva Ducke. Guia de identificação das plantas vasculares de uma floresta de terra-firme na Amazônia Central. Manaus, INPA-DFID.

StatSoft. 2003. Versão 6,0. East $14^{\text {th }}$ Street, Tulsa, StatSoft Inc.

Strasser, R.J.; Srivasatava, A.; Govindjee. 1995. Polyphasic chlorophyll a fluuorescence transient in plants and cyanobacteria, Photochemistry and Photobiology 61: 32-34.

Tundisi, J.G. 2007. Exploração do potencial hidrelétrico da Amazônia. Estudos Avançados 21(59): 109-117.

Waldhoff, D.; Furch, B. \& Junk, W.J. 2002. Fluorescence parameters, chlorophyll concentration, and anatomical features as indicators for flood adaptation of an abundant tree species in Central Amazonia: Symmeria paniculata. Environmental and Experimental Botany 48: 225-235.

Zanandrea, I.; Alves, J.D.; Deuner, S.; Goulart, P.F.P.; Henrique, P. C.; Silveira, N. M. 2009. Tolerance of Sesbania virgata plants to flooding. Australian Journal of Botany 57: 661-669. 